... Dominic Huser: Assistenzarzt Chirurgie am Luzerner Kantonsspital in Sursee und wahrscheinlich jüngster Arzt in der Schweiz

\title{
«Ich will Augen und Ohren offen halten»
}

\section{Daniel Lüthi}

Text und Bilder

danielluethi@gmx.ch
Wir treffen uns zwischen seinen ersten beiden Serien Notfalldienst, nach dem ersten Spätdienst. Bis am frühen Morgen schrieb Dominic Huser nach dem offiziellen Dienstende noch Berichte - in rund drei Stunden geht's wieder los, wie immer mit ungewissem Pensum. "Am Anfang hat man halt noch keine Routine, deshalb muss man am Schluss dann jeweils noch etwas länger bleiben», sagt einer, der sich als «Nachtmensch» bezeichnet und am Morgen jeweils ohnehin etwas Mühe hat mit dem Aufstehen.

\section{Der Überflieger}

Dominic Huser schloss sein Medizinstudium letzten September ab - im Alter von 22 Jahren. Damit ist er mit grosser Wahrscheinlichkeit der jüngste Arzt in der Schweiz. Der FMH ist eine Ärztin bekannt, die noch ein wenig jünger ist: Sie wurde im August 2010 21jährig und bestand im November das Staatsexamen in Lausanne. Wie auch immer: Huser reiste nach seinem Abschluss mit Kollegen in einem Camping-Bus nach Griechenland und dann mit seiner Mutter nach

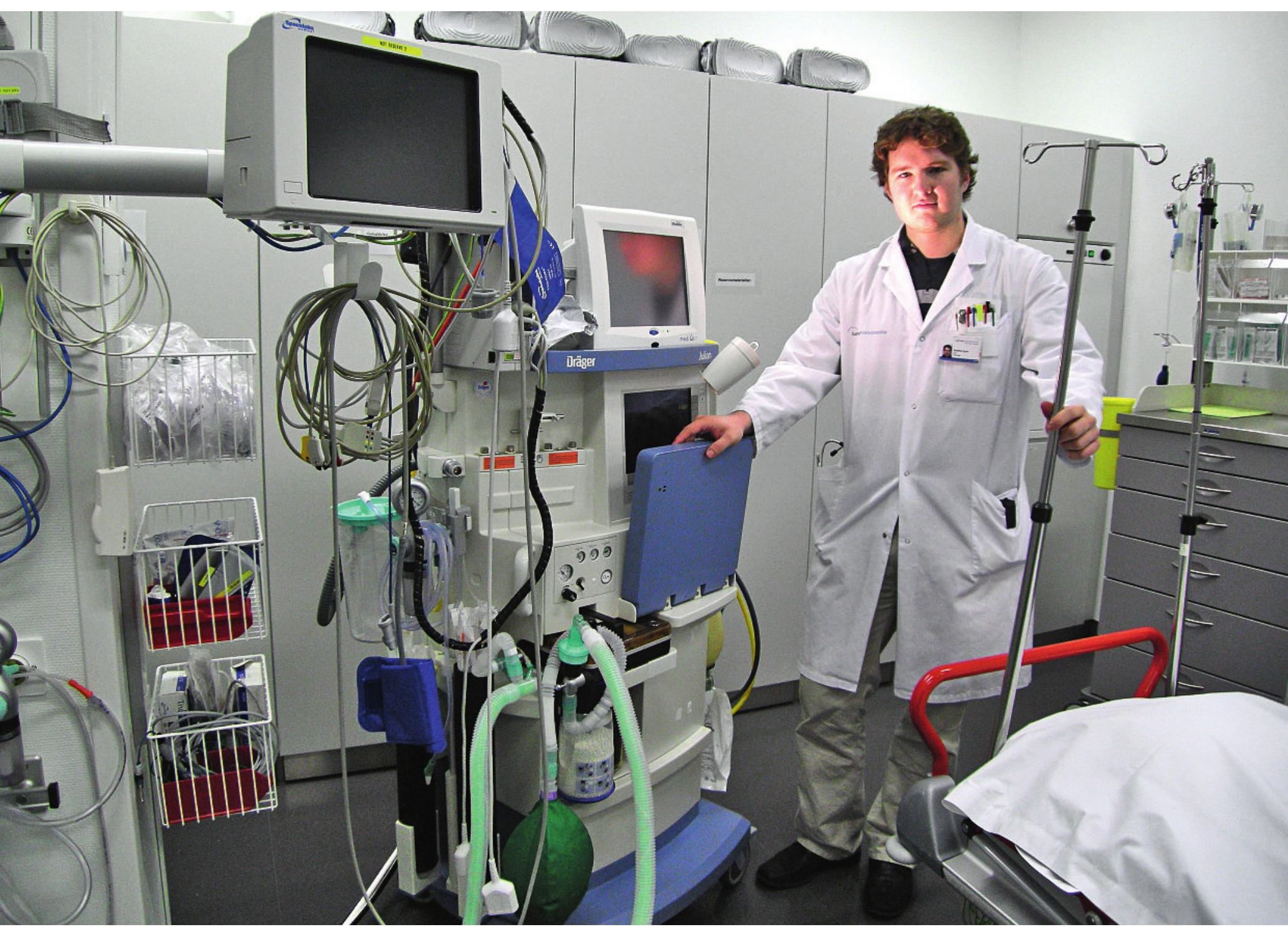


Hongkong und China, mit anschliessenden TauchTagen in Bali: «Super-Ferien», schwärmt er, «aber sie waren auch nötig.» Die Diplomfeier war Ende Oktober. Bis Ende Jahr schrieb er dann seine Doktorarbeit im Bereich Nuklearmedizin fertig. Um die Bildgebung bei Tumoren gehe es, erklärt er, um die Verlaufskontrolle bei Krebspatienten mittels radioaktiv markierter Stoffe - oder konkreter: um die "Positronen-Emissions-Tomografie PET».

Kaum zu glauben: Der junge Mann spricht mit der gleichen Selbstverständlichkeit über Tumore wie über Campingferien. Kaum zu glauben, dass einer in diesem Alter schon Arzt und schon so abgeklärt sein kann.

\section{«Meine Begabung machte mir mein Leben manchmal auch schwer.»}

Der Grund dafür wurde schon sehr früh erkannt, nämlich in der ersten oder zweiten Klasse, als bei Dominic Huser überdurchschnittliche Begabungen diagnostiziert wurden. Er ist seiner damaligen Lehrerin und den involvierten Psychologen immer noch dankbar für ihre Abklärungen und Folgerungen, denn die Klarheit, die damit geschaffen wurde, erleichterte sein Leben insgesamt, auch wenn sie gelegentlich für Schwierigkeiten sorgte. Tatsache ist: Dominic Huser ist ausserordentlich intelligent, deshalb konnte er in seiner Schulkarriere insgesamt drei Klassen überspringen. Und deshalb machte er seine Matura mit 16 Jahren und begann in diesem jugendlichen Alter bereits sein Medizinstudium. Sieht er sich deswegen als Glückspilz? «Ja, schon. Aber: Meine Begabung machte mir mein Leben manchmal auch schwer.» So wirkten sich erste Medienberichte über das «Wunderkind» in der Pubertät eher negativ aus, Huser wurde gehänselt, fühlte sich oft ausgestossen. «Ich musste nie dafür kämpfen, dass ich es schaffe, ich brauchte die Ellbogen-Mentalität nicht.» Das machte einsam. Und etwas träge. "Heute beeinträchtigt mich das alles nicht mehr.» Der Stempel des Speziellen und damit die «Vorurteils-Barriere», wie er sagt, sind in den Hintergrund getreten. Heute ist er Arzt - basta.

\section{Die Schlüsselerfahrung}

Erwachsen - auch beruflich erwachsen - wurde Dominic Huser vor allem wegen einer anderen Diagnose. Als er 19jährig und im vierten Studienjahr war, fand man bei seinem Vater einen Hirntumor, «etwas vom Schlimmsten, was man haben kann.» Vor drei Jahren starb er 54jährig in den Armen seines Sohnes. «In dieser Zeit lernte ich die Medizin von einer ganz anderen Seite kennen», erinnert sich Dominic Huser. Zum Beispiel: «Ich lernte warten, warten, warten.» Oder: «Ich lernte, dass man als Arzt keinen Knochenbruch, keinen Bauch und keinen Tumor behandelt.

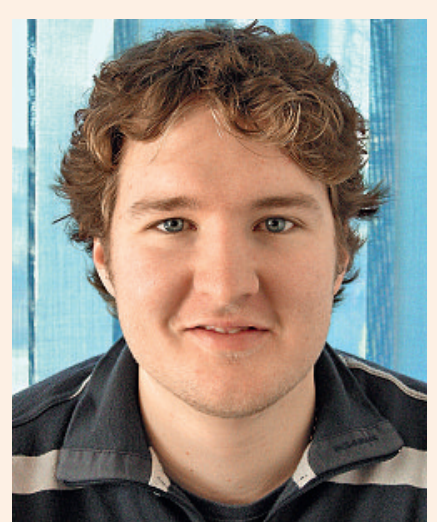

\section{Dominic Huser}

Dominic Huser wurde am 21. Januar 1988 in Kilchberg/Zürich geboren. Er wuchs in Rudolfstetten im Kanton Aargau auf und besuchte dort auch die Schulen. Weil er als überdurchschnittlich begabt galt, konnte er insgesamt drei Schulklassen überspringen. Mit 16 Jahren machte er in Wohlen/AG die Matura. Gleich anschliessend begann er an der Universität Zürich sein Medizinstudium, das er 2010 - im Alter von 22 Jahren - mit dem Staatsexamen abschloss. Seine Praktika führten ihn nach Chur (HNO), Aarau (Innere Medizin), St. Gallen (Radiologie), Zürich (Anästhesie), Olten (Allgemeine Chirurgie) und Boston in den USA (Neurochirurgie).

Seit Anfang Jahr arbeitet Dominic Huser als Assistenzarzt Chirurgie am Luzerner Kantonsspital in Sursee. Dort wohnt er im Personalhaus.

Sondern einen Patienten - einen Menschen. Deshalb gehören für mich Empathie und eigene Gefühle immer dazu. Ich hoffe, dass ich diese Einstellung nie verlieren werde.»

Eine Schlüsselerfahrung am Spitalbett seines Vaters war ein Phänomen aus dem Bereich «Kommunikation»: «Mehrmals erklärten Fachleute meinem Vater seine Krankheit und die wahrscheinlichen Folgen, sie machten das gut und er hörte zu, aber er erfasste den Ernst der Lage nicht. Ich verstand - er nicht. Vielen Ärzten ist wohl nicht bewusst, wie stark 
das Verständnis von Patienten in solchen Situationen beeinträchtigt sein kann.»

Bewusst wurden Dominic Huser damals weitere entscheidende Dinge, die ihn wohl während seiner ganzen Karriere begleiten werden: «Sehr früh erlebte ich die Grenzen des Machbaren.» Denn: «Ein Patient heilt sich selber. Ich kann ihn in diesem Prozess bloss unterstützen. Oft geht es nur darum, zu spüren, mitzuleben, Ohnmacht auszuhalten. Als Arzt darf man sich deshalb nicht zu wichtig nehmen.» Ja, so spricht ein 23-Jähriger, der sich vorgenommen hat, für Dinge, die ihn das Leben lehrt, empfänglich zu

\section{«Sehr früh erlebte ich die Grenzen des Machbaren.»}

bleiben und sein eigenes Handeln immer wieder kritisch zu hinterfragen.

«Ich will Augen und Ohren offen halten», sagt Huser auch, wenn es um seine berufliche Zukunft geht: «Wer zu früh plant, vergibt Chancen. Heute weiss ich noch nicht, wohin mich mein Weg führen wird. Ich gebe mir die Zeit, bis ich mehr weiss.» Klar ist ihm jetzt schon dies: «Ich möchte nicht nur für den Beruf leben. Mein Vater machte das, und es ging ihm nicht nur gut dabei.»

\section{Der Alltag}

Anschliessend an die erwähnte Doktorarbeit und die Erfahrungen mit der Krankheit des Vaters - ist da Onkologie ein denkbares, späteres Spezialgebiet? Wohl eher nicht: «Das ist ein frustrierendes Gebiet, das einem die Grenzen der Medizin leider sehr bald aufzeigt. Die durchschnittliche Lebenszeit eines Patienten um ein paar wenige Monate zu verlängern, der dafür durch eine Hölle von Operationen und Chemotherapien gehen muss - diese Gratwanderung zwischen der Wahrung verschiedener Interessen finde ich sehr schwierig.» Und jetzt spricht Dominic Huser zum ersten Mal auch von eigenen Ängsten: «Der Tod gehört zum Leben, davor habe ich keine Angst. Ich habe Angst davor, meinen ersten eigenen Patienten im Zustand zu sehen, in dem sich mein Vater kurz vor seinem Tod befand. Ich weiss nicht, was das in mir auslösen kann.»
Nun, vorderhand geht es in diesem Alltag um Handfesteres. Verkehrsunfälle und Nierensteine. Und das Umschalten von der Theorie der Universität in die Praxis am Spital. Der junge Assistenzarzt sagt es so: «Hier kommt die Erfahrung zum Tragen, die mir noch fehlt. Oft sind es kleine, praktische Dinge, an denen ich häufig noch scheitere.» Aber er merkt und schätzt die kleinen Fortschritte - und das Klima im Team, das Zusammenspiel zwischen Medizin und Pflege und Patientin und Physiotherapie und Angehörigen und so weiter. «Als Assistenzarzt bin ich eine Art Schaltstelle. Hier lerne ich viel. Am meisten aber lerne ich aus Fehlern, die in diesem Beruf vielfach gefährliche Folgen haben können, das ist die Krux.» Und noch etwas lernt der junge Arzt in seiner ersten Stelle: Dass er die Zeit, die er dem einzelnen Patienten gerne widmen würde, in aller Regel nicht hat.

\section{Die Zukunft}

Sein Wissen weitergeben: Das ist etwas, was Huser seit jeher fasziniert. Während der Schulzeit gab er Nachhilfeunterricht, im Studium leitete er Tutorien und in einer Kosmetik- und Massage-Schule vermittelte er medizinische Grundlagen. Vielleicht liegt die $\mathrm{Zu}-$ kunft in der Lehre? Wie auch immer: Auf sein eigenes Medizinstudium zurückblickend bedauert Dominic Huser, dass hier akademische Ansätze wünschbare praktische Teile allzu sehr verdrängen: «Ich finde es schade, dass man bei der Reform des Studiums die Chance verpasst hat, die Studenten besser auf das klinische Denken und Handeln vorzubereiten. Anstelle von praxisbezogenem Know-how steht akademisches Detailwissen immer noch zu stark im Vordergrund. Die Medizin als Kunst und Handwerk kommt so im Studium leider zu kurz.»

Daran, Standespolitiker zu werden, denkt Dominic Huser trotz solch grundsätzlicher Überlegungen vorerst nicht, dazu fühlt er sich noch zu unerfahren, «dafür habe ich noch zu wenig Ideen.»

Zur Zeit denkt er in den kurzen Pausen, die seine langen und intensiven Tage und Wochen im Spital erlauben, manchmal an etwas ganz anderes: ans Fischen im nahen Sempachersee. Das sei ähnlich meditativ wie die Arbeit im Operationssaal, schwärmt er, «da existiert die Aussenwelt nicht mehr, da kann ich ganz im Moment leben.»

Die Ausrüstung fürs Fischen hat er schon gekauft. Und das Brevet gemacht. Fehlt nur noch die Praxis.

Am Ende jeden Monats stellt die Schweizerische Ärztezeitung eine Persönlichkeit vor, die sich im Gesundheitswesen engagiert. Im Mai schildert Daniel Lüthi seine Begegnung mit Urban Laffer, Chefarzt Chirurgie am Spitalzentrum Biel und Präsident des Verbandes chirurgisch und invasiv tätiger Ärztinnen und Ärzte der Schweiz (fmCh). 